\title{
Cardiac Tests in Asymptomatic Type 2 Diabetics
}

\author{
Abdulnabi T. Al-Attara Suad A. Mahussain ${ }^{\mathrm{b}}$ S. Sadanandan ${ }^{\mathrm{c}}$ \\ aDiabetology Unit, ${ }^{b}$ Department of Nuclear Medicine, and cCardiology Unit, Al-Amiri Hospital, Ministry of Health, \\ Kuwait
}

\section{Key Words}

Diabetes mellitus - Myocardial ischemia $\cdot$ Cardiac exercise tolerance test . Myocardial perfusion scan . Echocardiography $\cdot$ Microalbuminuria

\begin{abstract}
Objective: To evaluate the use of noninvasive procedures for the detection of myocardial ischemia and its relation with other coexistent clinical factors in patients with asymptomatic type 2 diabetes mellitus. Subjects and Methods: $A$ total of 42 patients with type 2 diabetes mellitus, aged 41-72 years with no clinical history suggestive of coronary heart disease, were evaluated for silent myocardial ischemia by stress cardiac exercise tolerance test (ETT), 12-lead electrocardiography (ECG), transthoracic echocardiography and stress myocardial perfusion scan using technetium-99m tetrofosmin. Results: Eleven patients (26.2\%) showed an ischemic pattern on ETT, the resting ECG was suggestive of ischemia in only $2(4.8 \%)$, echocardiography showed diastolic dysfunction in 9 (21.4\%), and the stress myocardial perfusion scan was ischemic in $3(7.3 \%)$. For subjects over the age of 57, a significant difference was found between age and ischemic ETT ( $p=0.026)$ and diastolic dysfunction by echocardiography $(p=0.044)$. Patients with $\mathrm{mi}$ croalbuminuria and/or diastolic dysfunction were more
\end{abstract}

likely than others to have ischemic ETT ( $p=0.036$ and 0.024 , respectively) and patients with diastolic dysfunction had a higher prevalence of ischemic ETT. There was no relation between ischemic ETT and other major cardiac risk factors (hypertension, dyslipidemia, smoking, sex, duration of diabetes, BMI, and glycated hemoglobin levels). Conclusion: The cardiac ETT was most helpful for detecting myocardial ischemia in asymptomic type 2 diabetics. For equivocal ETT findings, echocardiography is recommended. The prevalence of myocardial ischemia was high in patients with type 2 diabetes mellitus.

Copyright @2002 S. Karger AG, Basel

\section{Introduction}

Diabetes mellitus type 2 (T2DM) is a growing worldwide medical problem. It is estimated that 135 million people were affected in 1995, and by the year 2025, the number is expected to reach 300 million [1]. The prevalence of T2DM in Kuwait is $14.8 \%$ [2] and increasing, placing Kuwait among other countries with a high prevalence of diabetes. The T2DM increases the risk of coronary heart disease by a factor of 2-4 [3], and patients with type 2 diabetes who have not had a myocardial infarction have a risk of infarction similar to that among nondiabetic patients who have had a prior myocardial infarction

\begin{tabular}{ll}
\hline KARGER & ( ) 2002 S. Karger AG, Basel \\
1011-7571/02/0114-0171\$18.50/0 \\
$\begin{array}{l}\text { Fax +4161306 1234 } \\
\begin{array}{l}\text { E-Mail karger@karger.ch } \\
\text { www.karger.com }\end{array}\end{array}$ & $\begin{array}{l}\text { Accessible online at: } \\
\text { www.karger.com } / \mathrm{mpp}\end{array}$
\end{tabular}

Dr. Abdulnabi Al-Attar
Diabetology Unit, Al-Amiri Hospital
Ministry of Health, Safat (Kuwait)
Tel. +9655335634, E-Mail Abdul_2345@yahoo.com 
[4]. Therefore, early detection of coronary heart disease in diabetics has become a priority, along with the need for coronary screening.

It is well known that coronary angiography is both highly sensitive and very specific in diagnosing coronary artery disease. However, this procedure cannot be used routinely for screening because it is an expensive and invasive procedure. Hence there is a need for a noninvasive cardiac screening test that has an acceptable sensitivity and specificity and is readily available and inexpensive.

Both the exercise tolerance test (ETT) and electrocardiography (ECG) are widely available, reasonably safe, relatively inexpensive and more importantly, of an acceptable sensitivity and specificity [5-7]. While echocardiography and myocardial perfusion imaging are noninvasive, they are more expensive than the ETT and ECG. The aim of this study was to evaluate the use of noninvasive procedures for the detection of myocardial ischemia in T2DM patients.

\section{Subjects and Methods}

A total of 42 T2DM patients who attended the diabetic outpatient clinic at Al-Amiri Hospital, Kuwait between February 2000 and January 2001 were included in the study. Twenty-six were males and 16 were females. The duration of the diabetes was $3-42$ years (12.9 \pm 8.4). The criteria for inclusion in this study were: a diagnosis of T2DM, age 41-72 years, no clinical history (past or present) suggestive of myocardial ischemia, and no current foot ulcer or other condition that could prevent the patient from performing a cardiac stress test. We primarily examined cardiac ETT profile and compared it to other commonly used, noninvasive cardiac testing methods: ECG, echocardiography, and myocardial perfusion imaging. The study was approved by the ethics committee of the hospital and informed consent was obtained from all the patients.

\section{Cardiac ETT}

Patients performed a treadmill stress test in accordance with the Bruce protocol. Heart rate, blood pressure and ECG were continuously recorded at baseline, during the exercise and into recovery. ST segment deviation was measured $80 \mathrm{~ms}$ after $\mathrm{J}$ point. The exercise was terminated when the patient developed angina pectoris or ST or QRS changes occurred.

\section{Transthoracic Echocardiography}

Two-dimensional and Doppler echocardiographic examinations were performed with an ATL HDI $3000 \mathrm{CV}$ machine using a 2.5$\mathrm{MHz}$ transducer. Examinations were performed in the left lateral supine position. Pulsed-wave Doppler studies of left ventricle filling were performed using the apical four-chamber view, with the sample volume positioned at the mitral leaflet tips, adjusted to produce maximal possible transmitral velocities using both the audible signal and the spectral velocity display. An ECG was simultaneously displayed.
Peak early diastolic flow velocity (E), peak late diastolic flow velocity due to atrial contraction (A), the ratio between peak early and peak late flow velocities (E/A ratio), acceleration and deceleration times of early diastolic velocity and duration of A wave were measured.

Pulmonary venous velocities were obtained by placing the sample volume $(0.5-1 \mathrm{~cm})$ in the right superior pulmonary vein in apical four-chamber view. Peak systolic (S) during ventricular systole, peak diastolic velocity (D), peak velocity and duration of reversal of flow (R) associated with atrial contraction (A) and the difference between the duration of the mitral A wave and pulmonary reversal wave were noted.

Isovolumic relaxation time, the interval between the end of ejection and onset of mitral inflow, was measured using continuous wave Doppler at an intermediate position between inflow and outflow to record both velocities. The diagnosis of diastolic dysfunction was made when an impaired left ventricular relaxation pattern was noted.

\section{Myocardial Perfusion Test}

Forty-eight hours prior to the test, all patients on beta-blockers and calcium channel antagonists were advised to stop the medications. The patients performed the treadmill exercise test using the standard Bruce protocol. At near-maximal exercise, a $20-$ to $25-\mathrm{mCi}$ dose of technetium- $99 \mathrm{~m}\left({ }^{99 \mathrm{~m}} \mathrm{Tc}\right)$ tetrofosmin was injected, after which exercise continued for $1 \mathrm{~min}$. Single photon emission computed tomography (SPECT) was begun 60 min after administration of the radiopharmaceutical.

Patients who had an abnormal stress myocardial perfusion scan were recalled 1-2 days later to do the rest myocardial perfusion scan generally known as the 2-day protocol, during which patients were reinjected intravenously with $20 \mathrm{mCi}{ }^{99} \mathrm{~m} \mathrm{Tc}$ tetrofosmin at rest. Rest SPECT imaging was initiated $90 \mathrm{~min}$ after the injection. The SPECT studies were performed which a circular orbit of $180^{\circ}$. Acquisition projections were 32 , with 25 s per projection. During imaging, a $20 \%$ window was centered on the $140-\mathrm{keV}$ peak of the ${ }^{99 \mathrm{~m}} \mathrm{Tc}$.

Two nuclear medicine specialists visually interpreted short axis, vertical long axis and horizontal long axis of the myocardial tomograms. Myocardial regions were classified as having mild, moderate or severe ischemia if the stress perfusion studies showed mild, moderate or severe perfusion abnormalities, respectively, and a refill of the ischemic areas during rest studies. However, myocardial scar was defined if perfusion abnormalities in the stress study persisted in the rest study.

\section{Data Analysis}

SPSS for Windows (Version 10; SPSS, Chicago) was used for data management and statistical analysis. Results were means \pm SD. Means were compared using the Student's test, and percentages were compared with the chi-square test. Differences with probability values $<0.05$ were considered significant.

\section{Results}

Clinical characteristics of the subjects are given in table 1 . No patient had clinical symptoms or signs of myocardial ischemia at the time of the study. However, major risk factors for ischemic heart disease were noted, such as 
systemic hypertension (62\%), dyslipidemia (50\%), and cigarette smoking (14.3\%). Further analysis of the major risk factors showed that $5 \%$ of the patients had no risk factors for ischemic heart disease other than diabetes, $38 \%$ had one additional risk factor, another $38 \%$ had two additional risk factors and $19 \%$ had three additional risk factors. Obesity was prevalent and body mass index (BMI) was $30 \pm 4.9 \mathrm{~kg} / \mathrm{m}^{2}$. Microalbuminuria $>73.2 \pm$ $116.3 \mu \mathrm{g} / 24 \mathrm{~h}$ was seen in $40.5 \%$ of the patients and found to be significantly associated with ischemic ETT ( $\mathrm{p}=$ 0.03 ). Retinopathy was found in $66.7 \%$ of the patients.

Twenty-five cardiac abnormalities revealed by the four techniques are shown in table 2. Of these, ETT showed an ischemic pattern in 11 patients (26\%). Transthoracic echocardiography showed diastolic dysfunction in 9 patients $(21 \%)$ and the stress myocardial perfusion scan revealed ischemia in $3(7.1 \%)$, all of which were reversible. Only 2 of the 42 patients (4\%) showed a pattern suggestive of ischemia on resting ECG: one with symmetrical $\mathrm{T}$-wave inversion in lead 1 and aVL and flat $\mathrm{T}$ wave in lead V6. The other patient showed a Q wave higher than $50 \%$ of the $\mathrm{R}$ wave in leads 3 and aVF.

Of the 11 patients with an ischemic pattern on ETT, 5 showed diastolic dysfunction on echocardiography, 2 had a stress myocardial perfusion defect, and 1 had a pattern suggestive of ischemia on resting ECG. However, ETT missed 7 cases of myocardial dysfunction that were seen using the other three techniques: echocardiography 4, stress myocardial perfusion scan 2, and ECG 1.

A comparison of patients with ischemic ETT and patients with a normal pattern showed that ischemic patients were more albuminuric than normal patients: $73.2 \pm 116.3$ vs. $9.0 \pm 71.4 \mu \mathrm{g} / \mathrm{l}(\mathrm{p}=0.03)$, and diastolic dysfunction was more prevalent in ischemic patients than normal patients: 45.5 vs. $12.9 \%(\mathrm{p}=0.024 \%)$. Patients over 57 years of age were more likely than normal subjects to have an ischemic pattern on ETT $(p=0.026)$ and diastolic dysfunction on echocardiography $(p=0.044)$.

\section{Discussion}

Myocardial ischemia, occurring more frequently in diabetics than nondiabetics, is a major complication encountered in the progressive course of diabetes mellitus $[9,10]$. Diabetics are screened routinely for expected complications such as retinopathy, nephropathy and peripheral vascular disease [12], but very little is done for silent myocardial ischemia, a complication which could be a terminal event in its first clinical presentation [13,
Table 1. Clinical characteristics of the 42 patients

\begin{tabular}{ll}
\hline Total number of patients & 42 \\
\hline Sex, M/F & $26 / 16$ \\
Age, years & $52.1 \pm 7.7$ \\
Hypertension $(\mathrm{n}=26)$ & $62 \%$ \\
Smoking $(\mathrm{n}=6)$ & $14.3 \%$ \\
Dyslipidemia $(\mathrm{n}=21)$ & $50 \%$ \\
Retinopathy $(\mathrm{n}=28)$ & $66.7 \%$ \\
HbA $_{\mathrm{Ic}}, \%$ & $9.7 \pm 2.4$ \\
Duration of diabetes, years & $12.9 \pm 8.4$ \\
BMI, kg/m ${ }^{2}$ & $30.0 \pm 4.9$ \\
Urinary microalbuminuria, $\mu \mathrm{g} / 1$ & $62.7 \pm 107$ \\
\hline Number of major risk factors $(R F)^{l}$ & \\
No RF $(\mathrm{n}=2)$ & $4.8 \%$ \\
$1 \mathrm{RF}(\mathrm{n}=16)$ & $38 \%$ \\
$2 \mathrm{RF}(\mathrm{n}=16)$ & $38 \%$ \\
3 RF $(\mathrm{n}=8)$ & $19 \%$ \\
\hline
\end{tabular}

Data are means \pm SD or percent of affected patients.

1 Major risk factors other than diabetes are hypertension, dyslipidemia and smoking.

14]. In this study, $26.5 \%$ of the patients were ischemic by ETT but none were symptomatic, reflecting the high prevalence of silent myocardial ischemia among diabetics, consistent with previous findings [15].

In this study, 25 patients had various myocardial dysfunctions. Though no single testing method detected all of the dysfunctions, ETT was most useful, followed by echocardiography, then myocardial perfusion scan. The least effective method was ECG. Despite the fact that ETT was the most helpful, it failed to detect cardiac abnormalities in 7 patients, indicating the need to use additional techniques, particularly when ETT findings are equivocal. In such cases, we suggest echocardiography be performed. 
Table 3. Clinical characteristics of patients with ischemic and normal ETT

\begin{tabular}{lcll}
\hline Data & Ischemic ETT & Normal ETT & p value \\
\hline Patients, n (\%) & $11(26.2 \%)$ & $31(73.8 \%)$ & - \\
Sex, M/F & $8 / 3$ & $18 / 13$ & NS \\
Age, years & $55.2 \pm 8.3$ & $50.8 \pm 7.1$ & $\mathrm{NS}$ \\
Hypertension, \% & 72.7 & 58.1 & $\mathrm{NS}$ \\
Smoking, \% & 18.2 & 12.9 & $\mathrm{NS}$ \\
Dyslipidemia, \% & 45.5 & 51.6 & $\mathrm{NS}$ \\
Retinopathy, \% & 27.3 & 35.5 & $\mathrm{NS}$ \\
HbA $_{\text {Ic }}$ & $8.9 \pm 2.4$ & $9.9 \pm 2.3$ & $\mathrm{NS}$ \\
Duration, years & $11.7 \pm 7.2$ & $13.2 \pm 8.9$ & $\mathrm{NS}$ \\
BMI, kg/m $^{2}$ & $28.2 \pm 4.0$ & $30.7 \pm 5.0$ & $\mathrm{NS}$ \\
Urinary microalbumin, $\mu \mathrm{g} / 1$ & $9.0 \pm 71.4$ & $73.2 \pm 116.3$ & 0.036 \\
\end{tabular}

Data are means \pm SD or percent of patients with normal and ischemic ETT.

Risk factors in this study included hypertension, dyslipidemia, cigarette smoking, age and microalbuminuria. Hypertension, dyslipidemia and cigarette smoking did not differ in patients with and without ischemic ETT, consistent with observations previously reported by Janand-Delenne et al. [16]. In this study, there was no significant difference in age between ischemic and nonischemic patients, which may reflect the increased incidence of atherosclerosis at early ages in diabetics [17]. Yet, patients over 57 years of age were more likely to have an ischemic pattern than those under 57 [18].

Since microalbuminuria without overt nephropathy is associated with a high cardiovascular mortality rate, cardiac stress testing is recommended [19]. In our study, the presence of microalbuminuria was highly associated with the presence of myocardial ischemia $(p=0.03)$, consistent with the findings of others $[15,20]$.

It is relatively easy to decide to investigate the diabetic patient presenting with angina pectoris or even the angina equivalent (exertional dyspnea, giddiness), but the real challenge is deciding on the best noninvasive cardiac testing method in asymptomatic patients with diabetes mellitus. ETT revealed the greatest number of cases $(n=11)$ of myocardial ischemia (table 3). However, echocardiography may be indicated for detecting silent myocardial ischemia among diabetics since diastolic dysfunction, common in diabetic patients [20,21], was found in $45.5 \%$ of the patients with ischemic ETT, significantly more frequently than in normal patients $(12.9 \%, p=0.024)$. In addition, cost and by extension, accessibility, must be considered, ECG being the least expensive followed by ETT, then echocardiography, with myocardial perfusion imaging the most expensive of all. Since ETT proved more helpful than any other testing method and is one of the least expensive techniques, it seems that ETT should be the noninvasive cardiac testing technique of choice when investigating asymptomatic diabetics for cardiac abnormalities.

We note that a potential limitation of our study is the relatively small number of our sample population.

\section{Conclusion}

The cardiac ETT was most helpful for detecting myocardial ischemia in asymptomic type 2 diabetics. For equivocal ETT findings, echocardiography is recommended. The prevalence of myocardial ischemia was high in the T2DM patients.

\section{References}

1 King H, Aubert RE, Herman WH: Global burden of diabetes, 1995-2025: Prevalence, numerical estimates, and projections. Diabetes Cre 1998;21:1414-1431.

2 Abdella N, Al Arouj M, Al Nakhi A, Al Assoussi A, Moussa M: Non-insulin-dependent diabetes in Kuwait: Prevalence rates and associated risk factors. Diabetes Res Clin Pract 1998; 42:187-196.

3 Haffner SM: Coronary heart disease in patients with diabetes. N Engl J Med 2000;342:10401042 .
4 Haffner SM: Mortality from coronary heart disease in subjects with type 2 diabetes and in nondiabetic subjects with and without prior myocardial infarction. N Engl J Med 2000;339: 229-234.

5 Lee TH, Boucher CA: Noninvasive test in patients with stable coronary artery disease. N Engl J Med 2001;344:1840-1845.

6 Rautaharju PM, Prineas RJ, Eifler WJ, Furberg CD, Neaton JD, Crow RS, Stamler J, Cutler JA: prognostic value of electrocardiogram in men at high risk of future coronary heart disease: Multiple risk factor intervention trial experience. J Am Coll Cardiol 1986;8:1-10.
7 Bruce RA, Hossack KF, DeRouen TA, Hofer $\mathrm{V}$ : Enhanced risk assessment for primary coronary heart disease events by maximal exercise testing: 10 years' experience of Seattle heart watch. J Am Coll Cardiol 1983;3:565-573.

8 Giannuzzi P, Imparato A, Temporelli PL: Doppler-derived mitral deceleration time of early filling as a strong predictor of pulmonary capillary wedge pressure in postinfarction patients with left ventricular systolic dysfunction. J Am Coll Cardiol 1994;23:1630-1637. 
9 Klein AL, Burstow DJ, Tajik J, Zachariah PK, Bailey KR, Seward JB: Effects of age on left ventricular dimensions and filling dynamics in 117 normal persons. Mayo Clin Proc 1994;69: 212-224.

10 Bloomgarden ZT: American Diabetes Association Annual Meeting, 1998: Cardiac disease and related topics. Diabetes Care 1998,21: 1764-1773.

11 Schurgin S, Rich S, Mazzone T: Increased prevalence of coronary artery calcification in patients with diabetes. Diabetes Care 2001;24: 335-338.

12 American Diabetic Association: Standard of Medical Care for Patients with Diabetes mellitus. Diabetes Care 2001;24:S33-S43.

13 Balkau B, Jouven X, Ducimetiere P, Eschwege $\mathrm{E}$ : Diabetes as a risk factor for sudden death. Lancet 1999;354:1968-1969.

14 Miettinen H, Lehto S, Salomaa V, Mahonen M, Niemela M, Haffner SM, Pyorala K, Tuomilehto J: Impact of diabetes on mortality after the first myocardial infarction. Diabetes Care 1998;21:69-75.
15 Mosca L, Grundy SM, Judelson D, King K, Limacher M, Oparil S, Pasternak R, Pearson TA, Redberg RF, Smith SC Jr, Winston M, Zinberg S: Guide to preventive cardiology for women. Circulation 1999;99:2480-2484.

16 Janand-Delenne B, Savin B, Habib G, Bory M, Vague $\mathrm{P}$, Lassmann-Vague V: Silent myocardial ischemia in patients with diabetes: Who to screen. Diabetes Care 1999;22:1396-1400.

17 Naka M, Hiramatsu K, Aizawa T, Momose A, Yoshizawa K, Shigematsu S, Ishihara F, Niwa A, Yamada T: Silent myocardial ischemia in patients with non-insulin-dependent diabetes mellitus as judged by treadmill exercise testing and coronary angiography. Am Heart J 1992; 123:46-53.

18 Cooper S, Caldwell JH: Coronary artery disease in people with diabetes: Diagnostic and risk factor evaluation. Clin Diabetes 1999;17: 58-70.
19 Biderman A, Rosenblatt I, Rosen S, Zangwill LM, Shalev R, Friger M, Weitzman S: Sex differentials in predictors of mortality for patients with adult-onset diabetes: A population-based follow-up study in Beer-Sheva, Israel. Diabetes Care 2000;23:602-605.

20 Mahgoub MA, abd-Elfattah AS: Diabetes mellitus and cardiac function. Mol Cell Biochem 1998;180:59-64.

21 Poirier P, Bogaty P, Garneau C, Marois L, Dumesnil J: Diastolic dysfunction in normotensive men with well-controlled type 2 diabetes. Diabetes Care 2001;24:5-10.

22 Detre KM, Lombardero MS, Brooks MM, Hardison RM, Richard H, Sopko G, Frye RL, Chaitman BR: The effect of previous coronary bypass surgery on the prognosis of patients with diabetes who have acute myocardial infarction. N Engl J Med 2000;342:989-997.

23 Barzilay JI, Spiekerman CF, Kuller LH, Burke GL, Bittner V, Gottdiener JS, Brancati FL, Orchard TJ, O'Leary DH, Savage PJ: Prevalence of clinical and isolated subclinical cardiovascular disease in older adults with glucose disorders. Diabetes Care 2001;24:1233-1239. 